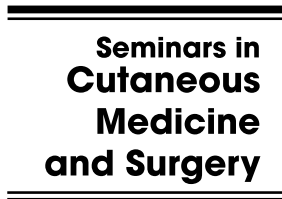

Volume 14, Number 4

September 2004

\title{
Something for Everyone!
}

$\mathrm{T}$ his edition of Seminars in Cutaneous Medicine and Surgery is a compilation of articles on a breadth of topics, reflecting the scope of our specialty. From cosmetic dermatology to medical dermatology to surgical dermatology, an outstanding group of contributors provide practical reviews of topics relevant to every day practice.

Dr. Leslie Baumann is widely respected for her expertise and innovation in cosmetic dermatology. Her article on fillers reviews a topic of great interest to us and to our patients. Dr. Baumann's thoughtful and careful review reflects the dermatologist's role at the forefront of these important therapies, and will bring you up to date on this rapidly evolving area.

Dermatologists are also at the vanguard of skin cancer management. Drs. Huang and Boyce provide a thorough analysis of surgical margins for management of non-melanoma skin cancer. Drs. McGillis and Fein review recent advances in the dermatologist's therapeutic arsenal in their discussion of medical management of skin cancer and precursor lesions.

Three of the articles might be best clustered under the topic Inside Mohs Surgery. Surgical pearls are prized by us all as a valuable means of enhancing our skills and efficiency. Dr. Albertini has assembled a special collection of valuable pearls gleaned from a busy Mohs practice. The nose is not only the most frequent site Mohs surgeons treat cancer, but is among the most challenging areas to reconstruct. Dr. Farley provides insight into principles of complex nasal reconstruction that is usually between the lines in textbooks and review articles. An article I co-authored with Dr. Wilde and my physician assistant Debbie Hauser reviews current issues and strategies relevant to the optimal and efficient management of surgical patients in the outpatient setting

Whether the focus of our practice is cosmetic, medical, or surgical dermatology, the vitality of our practice depends on the quality of service we provide our patients. The final article by Dr. Marks and colleagues is an insightful and important contribution on service excellence in Dermatology, and the principles eloquently discussed are of seminal importance to us all.

Barry Leshin, MD Guest Editor 